\title{
QPot: An R Package for Stochastic Differential Equation Quasi-Potential Analysis
}

by Christopher M. Moore, Christopher R. Stieha, Ben C. Nolting, Maria K. Cameron, and Karen C. Abbott

\begin{abstract}
QPot (pronounced $k y \overline{\mathbf{o} 0}+$ pät) is an R package for analyzing two-dimensional systems of stochastic differential equations. It provides users with a wide range of tools to simulate, analyze, and visualize the dynamics of these systems. One of QPot's key features is the computation of the quasi-potential, an important tool for studying stochastic systems. Quasi-potentials are particularly useful for comparing the relative stabilities of equilibria in systems with alternative stable states. This paper describes QPot's primary functions, and explains how quasi-potentials can yield insights about the dynamics of stochastic systems. Three worked examples guide users through the application of QPot's functions.
\end{abstract}

\section{Introduction}

Differential equations are an important modeling tool in virtually every scientific discipline. Most differential equation models are deterministic, meaning that they provide a set of rules for how variables change over time, and no randomness comes into play. Reality, of course, is filled with random events (i.e., noise or stochasticity). Unfortunately, many of the analytic techniques developed for deterministic ordinary differential equations are insufficient to study stochastic systems, where phenomena like noise-induced transitions between alternative stable states and metastability can occur. For systems subject to stochasticity, the quasi-potential is a tool that yields information about properties such as the expected time to escape a basin of attraction, the expected frequency of transitions between basins, and the stationary probability distribution. QPot (abbreviation of Quasi-Potential; Moore et al., 2016) is an R package that allows users to calculate quasi-potentials, and this paper is a tutorial of its application. This package is intended for use by any researchers who are interested in understanding how stochasticity impacts differential equation models. QPot makes quasi-potential analysis accessible to a broad range of modelers, including those who have not previously encountered the topic. The key functions in package QPot are listed in Table 1.

\section{Adding stochasticity to deterministic models}

Consider a differential equation model of the form

$$
\begin{aligned}
& \frac{d x}{d t}=f_{1}(x(t), y(t)) \\
& \frac{d y}{d t}=f_{2}(x(t), y(t)) .
\end{aligned}
$$

In many cases, state variables are subject to continuous random perturbations, which are commonly modeled as white noise processes. To incorporate these random influences, the original system of deterministic differential equations can be transformed into a system of stochastic differential equations:

$$
\begin{aligned}
& d X(t)=f_{1}(X(t), Y(t)) d t+\sigma d W_{1}(t) \\
& d Y(t)=f_{2}(X(t), Y(t)) d t+\sigma d W_{2}(t) .
\end{aligned}
$$

$X$ and $Y$ are now stochastic processes (a change emphasized through the use of capitalization); this means that, at every time $t, X(t)$ and $Y(t)$ are random variables, as opposed to real numbers. $\sigma \geq 0$ is a parameter specifying the noise intensity, and $W_{1}(t)$ and $W_{2}(t)$ are Wiener processes. A Wiener process is a special type of continuous-time stochastic process whose changes over non-overlapping time intervals, $\Delta t_{1}$ and $\Delta t_{2}$, are independent Gaussian random variables with means zero and standard deviations $\sqrt{\Delta t_{1}}$ and $\sqrt{\Delta t_{2}}$, respectively. The differential notation in equations (2) is a formal way of representing a set of stochastic integral equations, which must be used because realizations of Wiener processes are not differentiable (to be precise, with probability one, a realization of a Wiener process will be almost nowhere differentiable). The functions $f_{1}$ and $f_{2}$ are called the deterministic 


\begin{tabular}{|c|c|c|}
\hline Function & Main arguments & Description \\
\hline TSTraj() & $\begin{array}{l}\text { Deterministic skeleton, } \sigma \text {, } \\
T, \Delta t\end{array}$ & $\begin{array}{l}\text { Creates a realization (time series) of the } \\
\text { stochastic differential equations. }\end{array}$ \\
\hline TSPlot() & TSTraj() output & $\begin{array}{l}\text { Plots a realization of the stochastic dif- } \\
\text { ferential equations, with an optional his- } \\
\text { togram side-plot. Plots can additionally be } \\
\text { two-dimensional, which show realizations } \\
\text { in }(X, Y) \text {-space. }\end{array}$ \\
\hline TSDensity() & TSTraj() output & $\begin{array}{l}\text { Creates a density plot of a trajectory in } \\
(X, Y) \text {-space in one or two dimensions. }\end{array}$ \\
\hline QPotential() & $\begin{array}{l}\text { Deterministic skele- } \\
\text { ton, stable equilibria, } \\
\text { bounds, mesh (number of } \\
\text { divisions along each axis) }\end{array}$ & $\begin{array}{l}\text { Creates a matrix corresponding to a } \\
\text { discretized version of the local quasi- } \\
\text { potential function for each equilibrium. }\end{array}$ \\
\hline QPGlobal() & $\begin{array}{l}\text { Local quasi-potential ma- } \\
\text { trices, unstable equilibria }\end{array}$ & Creates a global quasi-potential surface. \\
\hline QPInterp() & $\begin{array}{l}\text { Global quasi-potential, } \\
(x, y) \text {-coordinates }\end{array}$ & $\begin{array}{l}\text { Evaluates the global quasi-potential at } \\
(x, y) \text {. }\end{array}$ \\
\hline QPContour() & Global quasi-potential & $\begin{array}{l}\text { Creates a contour plot of the quasi- } \\
\text { potential. }\end{array}$ \\
\hline VecDecomAll() & $\begin{array}{l}\text { Global quasi-potential, } \\
\text { deterministic skeleton, } \\
\text { bounds }\end{array}$ & $\begin{array}{l}\text { Creates three vector fields: the deter- } \\
\text { ministic skeleton, the negative gradient } \\
\text { of the quasi-potential, and the remain- } \\
\text { der vector field. To find each field in- } \\
\text { dividually, the functions } \operatorname{VecDecomVec}() \text {, } \\
\text { VecDecomGrad(), or } \operatorname{Vec} D e c o m R e m() \text { can be } \\
\text { used. }\end{array}$ \\
\hline VecDecomPlot() & $\begin{array}{l}\text { Deterministic skeleton, } \\
\text { gradient, or remainder } \\
\text { field }\end{array}$ & $\begin{array}{l}\text { Creates a vector field plot for the vector, } \\
\text { gradient, or remainder field. }\end{array}$ \\
\hline
\end{tabular}

Table 1: Key functions in package QPot.

skeleton. The deterministic skeleton can be viewed as a vector field that determines the dynamics of trajectories in the absence of stochastic effects. We will forgo a complete overview of stochastic differential equations here; interested readers are encouraged to seek out texts like Allen (2007) and Iacus (2009). We note that throughout this paper we use the Itô formulation of stochastic differential equations.

\section{The quasi-potential}

Consider System (2), with deterministic skeleton (1). If there exists a function $V(x, y)$ such that $f_{1}(x, y)=-\frac{\partial V}{\partial x}$ and $f_{2}(x, y)=-\frac{\partial V}{\partial y}$, then System (1) is called a gradient system and $V(x, y)$ is called the system's potential function. The dynamics of a gradient system can be visualized by considering the $(x, y)$-coordinates of a ball rolling on a surface specified by $z=V(x, y)$. Gravity causes the ball to roll downhill, and stable equilibria correspond to the bottoms of the surface's valleys. $V(x, y)$ is a Lyapunov function for the system, which means that if $(x(t), y(t))$ is a solution to System (1), then $\frac{d}{d t}(V(x(t), y(t))) \leq 0$, and the only places that $\frac{d}{d t}(V(x(t), y(t)))=0$ are at equilibria. This means that the ball's elevation will monotonically decrease, and will only be constant if the ball is at an equilibrium. The basin of attraction of a stable equilibrium $\mathbf{e}^{*}$ of System (1) is the set of points that lie on solutions that asymptotically approach $\mathbf{e}^{*}$.

The potential function is useful for understanding the stochastic System (2). As in the deterministic case, the dynamics of the stochastic system can be represented by a ball rolling on the surface $z=V(x, y)$; in the stochastic system, however, the ball experiences random perturbations due to noise terms in the System (2). In systems with multiple stable equilibria, these random perturbations can cause a trajectory to move between different basins of attraction. The depth of the potential (that is, the difference in $V$ at the equilibrium and the lowest point on the boundary of its basin of attraction), is a useful measure of the stability of the equilibrium (see Nolting and Abbott, 2016). The deeper the 
potential, the less likely it will be for stochastic perturbations to cause an escape from the basin of attraction. This relationship between the potential and the expected time to escape from a basin of attraction can be made precise (see formulae in the appendices of Nolting and Abbott, 2016). Similarly, the potential function is directly related to the expected frequency of transitions between different basins, and to the stationary probability distribution of System (2).

Unfortunately, gradient systems are very special, and a generic system of the form (1) will almost certainly not be a gradient system. That is, there will be no function $V(x, y)$ that satisfies $f_{1}(x, y)=-\frac{\partial V}{\partial x}$ and $f_{2}(x, y)=-\frac{\partial V}{\partial y}$.

Fortunately, quasi-potential functions generalize the concept of a potential function for use in nongradient systems. A non-gradient system's quasi-potential, $\Phi(x, y)$, possesses many of the properties of a gradient system's potential function; in particular, a non-gradient system's quasi-potential is related to its stationary probability distribution in the same way that a gradient system's potential function is related to its stationary probability distribution. Furthermore, $\Phi(x, y)$ is a Lyapunov function for the deterministic skeleton of a non-gradient system, just as a potential function is for a gradient system. Therefore, the surface $z=\Phi(x, y)$ is a highly useful stability metric. The quasipotential also provides information about the expected frequency of transitions between basins of attraction and the expected time required to escape each basin.

The mathematical definition of the quasi-potential is rather involved. We refer readers to Cameron (2012), Nolting and Abbott (2016), and the references therein for the technical construction.

In both this paper and in package QPot, the function that we refer to as the quasi-potential is $\frac{1}{2}$ times the quasi-potential as defined by Freidlin and Wentzell (2012). This choice is made so that the quasi-potential will agree with the potential in gradient systems.

QPot is an R package that contains tools for calculating and analyzing quasi-potentials (which, for the special case of gradient systems, are simply potentials). The following three examples show how to use the tools in this package. The first example is a simple consumer-resource model from ecology. This example is explained in detail, starting with the analysis of the deterministic skeleton, proceeding with simulation of the stochastic system, and finally demonstrating the calculation, analysis, and interpretation of the quasi-potential. The second and third examples are covered in less detail, but illustrate some special system behaviors. Systems with limit cycles, like Example 2, require a slightly different procedure than systems that only have point attractors. Extra care must be taken constructing global quasi-potentials for exotic systems, like Example 3.

\section{Example 1: A consumer-resource model with alternative stable states}

Consider the stochastic version (sensu (2)) of a standard consumer-resource model of plankton $(X)$ and their consumers (Y) (Collie and Spencer, 1994; Steele and Henderson, 1981):

$$
\begin{aligned}
& d X(t)=\left(\alpha X(t)\left(1-\frac{X(t)}{\beta}\right)-\frac{\delta X(t)^{2} Y(t)}{\kappa+X(t)^{2}}\right) d t+\sigma d W_{1}(t) \\
& d Y(t)=\left(\frac{\gamma X(t)^{2} Y(t)}{\kappa+X(t)^{2}}-\mu Y(t)^{2}\right) d t+\sigma d W_{2}(t)
\end{aligned}
$$

The model is formulated with a Type III functional response; the relationship between the plankton density and the per-capita consumption rate of plankton is sigmoid. $\alpha$ is the plankton's maximum population growth rate, $\beta$ is the plankton carrying capacity, $\delta$ is the maximal feeding rate of the consumers, $\gamma$ is the maximum conversion rate of plankton to consumer (which takes into account maximum feeding rate), $\kappa$ controls how quickly the consumption rate saturates, and $\mu$ is the consumer mortality rate. We will analyze this example with a set of parameter values that yield two stables states: $\alpha=1.54, \beta=10.14, \gamma=0.476, \delta=1, \kappa=1$, and $\mu=0.112509$.

\section{Step 1: Analyzing the deterministic skeleton}

There are preexisting tools in R for analyzing the deterministic skeleton of System (3), which will be described briefly in this subsection. Many of the these tools can be found in the CRAN Task View for Differential Equations (https://CRAN.R-project.org/view=DifferentialEquations), but we use a select few in our analysis. The first step is to find the equilibria for the system and determine their stability with linear stability analysis. Equilibria can be found using the package rootSolve (Soetaert and Herman, 2008). rootSolve provides routines that allow users to find roots of nonlinear functions, and perform equilibria and steady-state analysis of ordinary differential equations (ODEs). In Example 1 , the equilibria are $\mathbf{e}_{u 1}=(0,0), \mathbf{e}_{s 1}=(1.4049,2.8081), \mathbf{e}_{u 2}=(4.2008,4.0039), \mathbf{e}_{s 2}=(4.9040,4.0619)$, and $\mathbf{e}_{u 3}=(10.14,0)$. Eigenvalues of the linearized system at an equilibrium can be found by using 
eigen() in package base over the Jacobian matrix (jacobian. full() in package rootSolve), which determines the asymptotic stability of the system. $\mathbf{e}_{u 1}$ is an unstable source and $\mathbf{e}_{u 2}$ and $\mathbf{e}_{u 3}$ are saddles. The eigenvalues corresponding to $\mathbf{e}_{s 1}$ are $-0.047 \pm 0.458 i$ and the eigenvalues corresponding to $\mathbf{e}_{s 2}$ are -0.377 and -0.093 . Hence $\mathbf{e}_{s 1}$ is a stable spiral point and $\mathbf{e}_{s 2}$ is a stable node. To ease transition from packages such as deSolve (Soetaert et al., 2010) and rootSolve to our package QPot, we include the wrapper function Model2String(), which takes a function containing equations and a list of parameters and their values, and returns the equations in a string that is usable by QPot (see the help page for an example).

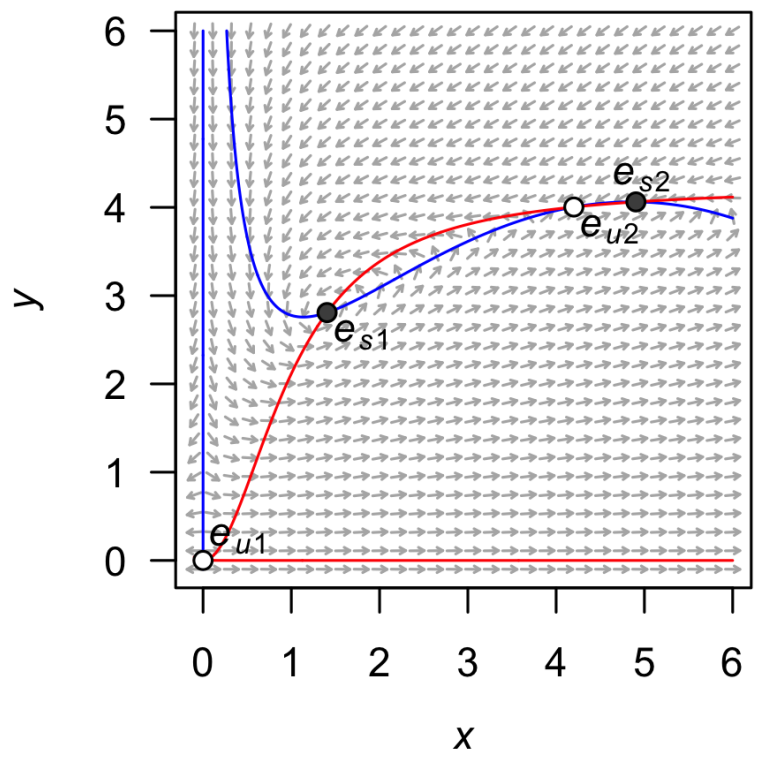

Figure 1: A stream plot of the deterministic skeleton of System (3). The blue line is an $x$-nullcline (where $\frac{d x}{d t}=0$ ) and the red line is a $y$-nullcline (where $\frac{d y}{d t}=0$ ). Open circles are unstable equilibria and filled circles are stable equilibria. Made using the package phaseR.

The package phase R (Grayling, 2014a,b) is an R package for the qualitative analysis of one- and two-dimensional autonomous ODE systems using phase plane methods (including the linear stability analysis described in the preceding paragraph). We use phase $R$ to generate a stream plot of the deterministic skeleton of System (3) (Figure 1). Note that a stream plot is a phase plane plot that displays solutions of a system of differential equations; these solutions are also called streamlines. The deSolve package can be used to find solutions corresponding to particular initial conditions of the deterministic skeleton of System (3). During the analysis of the deterministic skeleton of a system, it is important to note several things. The first is the range of $x$ and $y$ values over which relevant dynamics occur. In Example 1, transitions between the stable equilibria are a primary point of interest, so one might wish to focus on a region like the one displayed in Figure 1, even though this region excludes $\mathbf{e}_{u 3}$. The regions of phase space that the user finds interesting will determine the window sizes and ranges used later in the quasi-potential calculations. Second, it is important to note if there are any limit cycles. If there are, it will be necessary to identify a point on the limit cycle. This can be accomplished by calculating a long-time solution of the system of ODEs to obtain a trajectory that settles down on the limit cycle (see Example 2). Finally, it is important to note regions of phase space that correspond to unbounded solutions. As explained in subsequent sections, it is worth examining system behavior in negative phase space, even in cases where negative quantities lack physical meaning.

\section{Step 2: Stochastic simulation}

QPot contains several tools for generating and visualizing realizations of systems of the form (2). Examining these realizations can help users understand qualitative features of the system before computing and analyzing the quasi-potential. Sim.DiffProc (Guidoum and Boukhetala, 2016) and yuima (Brouste et al., 2014) are two packages that offer a full suite of stochastic differential equation simulation options, and many of the tools that they contain are more efficient than those in QPot. Users interested in very large-scale simulation are encouraged to seek out those packages. For general exploration of a model's behavior prior to quasipotential analysis, however, $\operatorname{TSTraj}()$ is extremely helpful and does not require the use of a separate package.

Here, we show how to use QPot to obtain realizations of System (3) for a specified level of noise intensity, $\sigma$. To do this, TSTraj() in QPot implements the Euler-Maruyama method. All other 
negative population densities are not physically meaningful. A lower bound can be imposed on the functions described in this subsection with the argument lower. bound in the function TSTraj(). Similarly, it might be desirable to set an upper bound for realizations, and hence prevent runaway trajectories (unbounded population densities are also not physically meaningful). An upper bound can be imposed on the functions described in this subsection with the argument upper. bound.
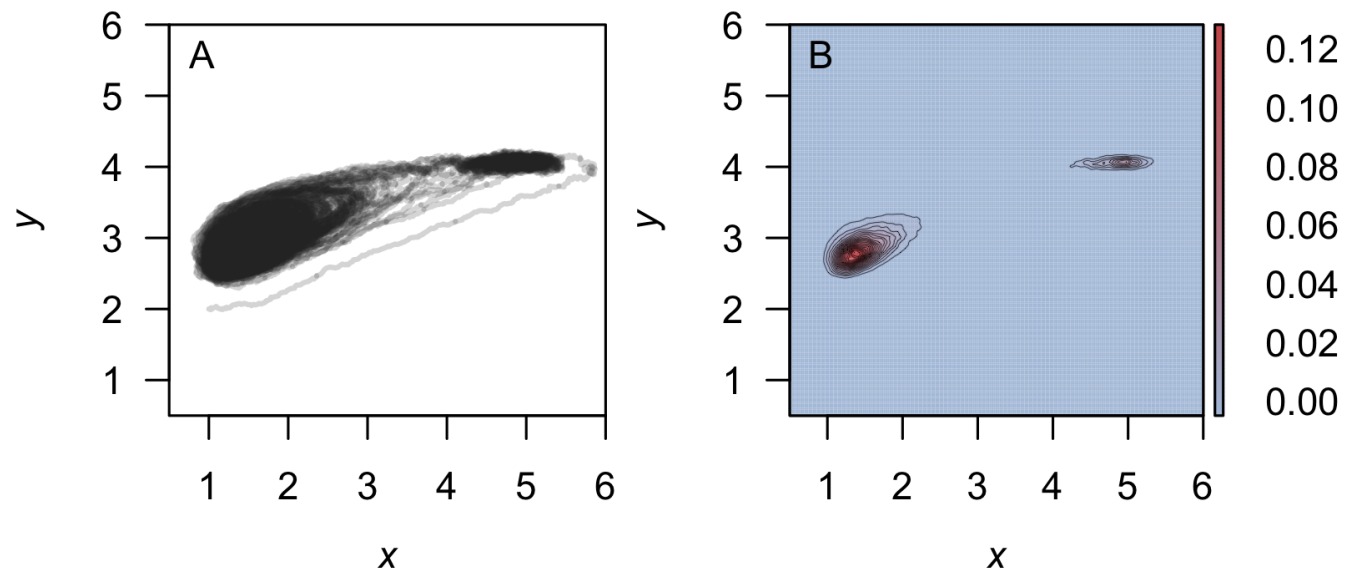

Figure 3: (A) The realization of System (3) created using TSPlot () plotted in $(x, y)$-space with dim = 2. (B) A density plot obtained from a realization of System (3) using the function TSDensity() with $\operatorname{dim}=2$. Red corresponds to high density, and blue to low density.

\section{Step 3: Local quasi-potential calculation}

The next step is to compute a local quasi-potential for each attractor. Because QPot deals with twodimensional systems, attractor will be used synonymously with stable equilibrium or stable limit cycle. A limit cycle will be considered in example 2. For now, suppose that the only attractors are stable equilibrium points, $\mathbf{e}_{s i}, i=1, \ldots, n$. In the example above, $n=2$. For each stable equilibrium $\mathbf{e}_{s i}$, we will compute a local quasi-potential $\Phi_{i}(x, y)$.

In order to understand the local quasi-potential, it is useful consider the analogy of a particle traveling according to System (2). In the context of Example 1, the coordinates of the particle correspond to population densities, and the particle's path corresponds to how those population densities change over time. The deterministic skeleton of (2) can be visualized as a force field influencing the particle's trajectory. Suppose that the particle moves along a path from a stable equilibrium $\mathbf{e}_{s i}$ to a point $(x, y)$. If this path does not coincide with a solution of the deterministic skeleton, then the stochastic terms must be doing some work to move the particle along the path. The more work is required, the less likely it is for the path to be a realization of System (2). $\Phi_{i}(x, y)$ is the amount of work required to traverse the easiest path from $\mathbf{e}_{s i}$ to $(x, y)$. Note that $\Phi_{i}(x, y)$ is non-negative, and it is zero at $\mathbf{e}_{s i}$.

In the basin of attraction for $\mathbf{e}_{s i}, \Phi_{i}(x, y)$ has many properties analogous to the potential function for gradient systems. Key among these properties is that the quasi-potential is non-increasing along deterministic trajectories. This means that the quasi-potential can be interpreted as a type of energy surface, and the rolling ball metaphor is still valid. The difference is that, in non-gradient systems, there is an additional component to the vector field that causes trajectories to circulate around level sets of the energy surface. This is discussed in more detail in Step 6, below.

QPot calculates quasi-potentials using an adjustment developed by Cameron (2012) to the ordered upwind algorithm (Sethian and Vladimirsky, 2001, 2003). The idea behind the algorithm is to calculate $\Phi_{i}(x, y)$ in ascending order, starting with the known point $\mathbf{e}_{s i}$. The result is an expanding area where the solution is known.

Calculating $\Phi_{i}(x, y)$ with the function QPotential() requires a text string of the equations and parameter values, the stable equilibrium points, the computation domain, and the mesh size. If the equations do not contain the parameter values, the function Model2String() can be used to insert the values into the equations, as presented above. For (3), this first means inputting the equations:

$$
\begin{aligned}
& f_{1}(x, y)=1.54 x\left(1-\frac{x}{10.14}\right)-\frac{x^{2} y}{1+x^{2}} \\
& f_{2}(x, y)=\frac{0.476 x^{2} y}{1+x^{2}}-0.112509 y^{2}
\end{aligned}
$$


In R:

\#\# If not done in a previous step.

parms.eqn. $x<-$ Model2String (var.eqn. $x$, parms $=$ model. parms)

\#\# Do not print to screen.

parms.eqn.y <- Model2String(var.eqn.y, parms = model. parms, supress.print $=$ TRUE)

\#\# Could also input the values by hand and use this version.

\#\# parms.eqn. $x<-" 1.54 * x *(1.0-(x / 10.14))-\left(y *\left(x^{\wedge} 2\right)\right) /\left(1.0+\left(x^{\wedge} 2\right)\right) "$

\#\# parms.eqn. $y<-"\left(\left(0.476 *\left(x^{\wedge} 2\right) * y\right) /\left(1+\left(x^{\wedge} 2\right)\right)\right)-0.112509 *\left(y^{\wedge} 2\right) "$

The coordinates of the points $\mathbf{e}_{s i}$, which were determined in Step 1 , are $\mathbf{e}_{s 1}=(1.4049,2.8081)$ and $\mathbf{e}_{s 2}=(4.9040,4.0619)$.

eq1.x $<-1.40491$

eq1.y $<-2.80808$

eq2. $x<-4.9040$

eq2. $y<-4.06187$

Next, the boundaries of the computational domain need to be entered. This domain will be denoted by $\left[L x_{1}, L x_{2}\right] \times\left[L y_{1}, L y_{2}\right]$. The ordered-upwind method terminates when the solved area encounters a boundary of this domain. Thus, it is important to choose boundaries carefully. For example, if $\mathbf{e}_{s i}$ lies on one of the coordinate axes, one should not use that axis as a boundary because the algorithm will immediately terminate. Instead, one should add padding space. This is important even if the padding space corresponds to physically unrealistic values (e.g., negative population densities). For this example, a good choice of boundaries is: $L x_{1}=L y_{1}=-0.5$, and $L x_{2}=L y_{2}=20$. This choice of domain was obtained by examining stream plots of the deterministic skeleton and density plots of stochastic realizations (Figures 1-3). The domain contains all of the deterministic skeleton equilibria, and it encompasses a large area around the regions of phase space visited by stochastic trajectories (Figures 1-3). Note that a small padding space was added to the left and bottom sides of the domain, so that the coordinate axes are not the domain boundaries.

bounds. $x<-c(-0.5,20.0)$

bounds.y $<-c(-0.5,20.0)$

In some cases, it may be desirable to treat boundaries differently in the upwind algorithm. This is addressed below in Section 6.7.

Finally, the mesh size for the discretization of the domain needs to be specified. Let $N_{x}$ be the number of grid points in the $x$-direction and $N_{y}$ be the number of grid points in the $y$-direction. Note that the horizontal distance between mesh points is $h_{x}=\frac{L x_{2}-L x_{1}}{N_{x}}$, and the vertical distance between mesh points is $h_{y}=\frac{L y_{2}-L y_{1}}{N_{y}}$. Mesh points are considered adjacent if their Euclidean distance is less than or equal to $h=\sqrt{h_{x}^{2}+h_{y}^{2}}$. This means that diagonal mesh points are considered adjacent. In this example, a good choice is $N_{x}=N_{y}=4100$. This means that $h_{x}=h_{y}=0.005$, and $h \approx 0.00707$. In general, the best choice of mesh size will be a compromise between resolution and computational time. The mesh size must be fine enough to precisely track how information moves outward along characteristics from the initial point. Too fine of a mesh size can lead to very long computational times, though. The way that computation time scales with grid size depends on the system under consideration (see below for computation time for this example), because the algorithm ends when it reaches a boundary, which could occur before the algorithm has exhaustively searched the entire mesh area.

step.number. $x<-1000$

step.number.y $<-1000$ \# we used 4100 in the figures

The update radii factors, $K_{x}$ and $K_{y}$, are two other adjustable parameters for the algorithm. These are k. $x$ and k. y in QPotential(). These two parameters determine the neighborhood of points that can be used to update a given point. $K_{x}$ and $K_{y}$ are the distances (measured in mesh units) in the $x$ and $y$ direction that bound this neighborhood for any given point. The selection of the best values for these parameters involves several nuanced considerations. For a discussion of these issues, please see Cameron (2012). For users who wish to avoid these details, we suggest using the defaults $K_{x}=20$ and $K_{y}=20$.

The R interface implements the QPotential() algorithm using C code. By default QPotential() outputs a matrix that contains the quasi-potentials to the $\mathrm{R}$ session. The time required to compute the quasi-potential will depend on the size of the region and the fineness of the mesh. This example with $K_{x}=K_{y}=20$ and $N_{x}=N_{y}=4100$ has approximately $1.7 \times 10^{7}$ grid points, which leads to run times of approximately $2.25 \mathrm{~min}(2.5 \mathrm{GHz}$ Intel Core i5 processor and 8 GB $1600 \mathrm{MHz}$ DDR3 memory). When one reaches around $5 \times 10^{8}$ grid points, computational time can be several hours. 
Setting the argument save. to. R to TRUE (default) outputs the matrix into the $\mathrm{R}$ session, and setting the argument save. to. HD to TRUE saves the matrix to the hard drive as a tab-delimited text file filename in the current working directory. For $N_{x}=N_{y}=4100$, the saved file occupies $185 \mathrm{MB}$.

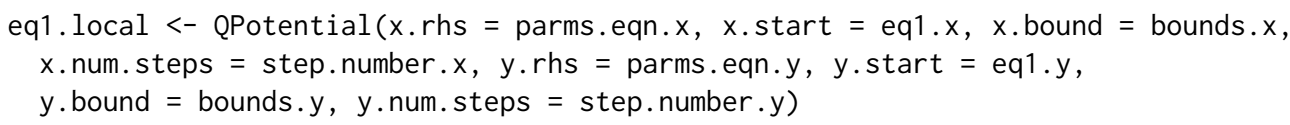

Step 3 should be repeated until local quasi-potentials $\Phi_{i}(x, y)$ have been obtained for each $\mathbf{e}_{s i}$. In Example 1, this means calculating $\Phi_{1}(x, y)$ corresponding to $\mathbf{e}_{s 1}$ and $\Phi_{2}(x, y)$ corresponding to $\mathbf{e}_{s 2}$.

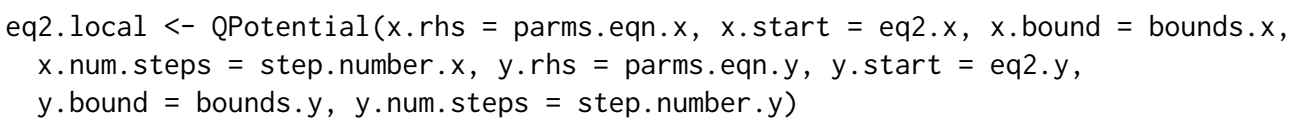

Each local quasi-potential $\Phi_{i}(x, y)$ is stored in $\mathrm{R}$ as a large matrix. The entries in this matrix are the values of $\Phi_{i}$ at each mesh point. To define the function on the entire domain (i.e., to allow it to be evaluated at arbitrary points in the domain, not just the discrete mesh points), bilinear interpolation is used. The values of $\Phi(x, y)$ can be extracted using the function QPInterp(). Inputs to QPInterp() include the $(x, y)$ coordinates of interest, the $(x, y)$ domain boundaries, and the QPotential() output (i.e., the matrix with rows corresponding to $x$-values and columns corresponding to $y$-values). QPInterp() can be used for any of the local quasi-potential or the global quasi-potential surfaces (see the next subsection).

\section{Step 4: Global quasi-potential calculation}

Recall that $\Phi_{i}(x, y)$ is the amount of work required to travel from $\mathbf{e}_{s i}$ to $(x, y)$. This information is useful for considering dynamics in the basin of attraction of $\mathbf{e}_{s i}$. In many cases, however, it is desirable to define a global quasi-potential that describes the system's dynamics over multiple basins of attraction. If a gradient system has multiple stable states, the potential function provides an energy surface description that is globally valid. We seek an analogous global function for non-gradient systems. Achieving this requires pasting local quasi-potentials into a single global quasi-potential. If the system has only two attractors, one can define a global quasi-potential, though it might be nontrivial (see Example 3 ahead). In systems with three or more attractors such a task might not be possible (Freidlin and Wentzell, 2012). For a wide variety of systems, however, a relatively simple algorithm can accomplish the pasting (Graham and Tél, 1986; Roy and Nauman, 1995). In most cases, the algorithm amounts to translating the local quasi-potentials up or down so that they agree at the saddle points that separate the basins of attraction. In Example 1, $\mathbf{e}_{u 1}$ lies on the boundary of the basins of attraction for $\mathbf{e}_{s 1}$ and $\mathbf{e}_{s 2}$. Creating a global quasi-potential requires matching $\Phi_{1}$ and $\Phi_{2}$ at $\mathbf{e}_{u 2} . \Phi_{1}\left(\mathbf{e}_{u 2}\right)=0.007056$ and $\Phi_{2}\left(\mathbf{e}_{u 2}\right)=0.00092975$. If one defines

$$
\Phi_{2}^{*}(x, y)=\Phi_{2}(x, y)+(0.007056-0.00092975)=\Phi_{2}(x, y)+0.00612625,
$$

then $\Phi_{1}$ and $\Phi_{2}^{*}$ match at $\mathbf{e}_{u 2}$. Finally, define

$$
\Phi(x, y)=\min \left(\Phi_{1}(x, y), \Phi_{2}^{*}(x, y)\right),
$$

which is the global quasi-potential. For systems with more than two stable equilibria, this process is generalized to match local quasi-potentials at appropriate saddles. QPot automates this procedure. A fuller description of the underlying algorithm is explained in Example 3, which requires a more nuanced understanding of the pasting procedure.

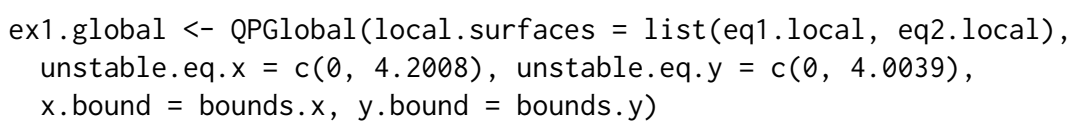

This function QPGlobal() calculates the global quasi-potential by automatically pasting together the local quasi-potentials. This function requires the input of all the discretized local quasi-potentials, and the coordinates of all unstable equilibria. The output is a discretized version of the global quasipotential. The length of time required for this computation will depend on the total number of mesh points; for the parameters used in Example 1, it takes a couple of minutes. As with the local quasi-potentials, the values of $\Phi(x, y)$ can be extracted using the function QPInterp().

\section{Step 5: Global quasi-potential visualization}

To visualize the global quasi-potential, one can simply take the global quasi-potential matrix from QPGlobal() and use it to create a contour plot using QPContour() (Figure 4). 

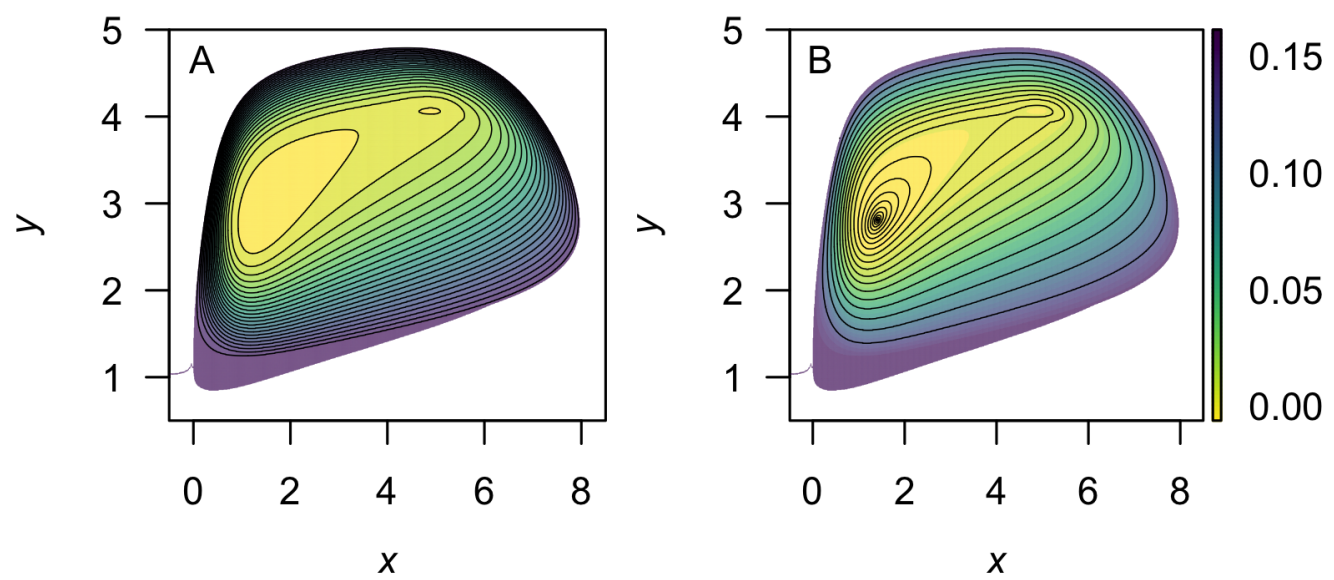

Figure 4: A contour plot of the of the quasi-potential of System (3). Yellow corresponds to low values of the quasi-potential, and purple to high values. The c. parm parameter in QPContour () can be used to generate non-equal contour spacing (e.g., for finer resolution near equilibria). The default creates evenly spaced contour lines ((A); c. parm $=1)$. In (B), contour lines are concentrated at the bottom of the basin (c. parm = 5). Default plot colors are generated from package viridis (Garnier, 2016), by setting col. contour $=\operatorname{viridis}(n=25$, option $=$ "D") in QPContour () .

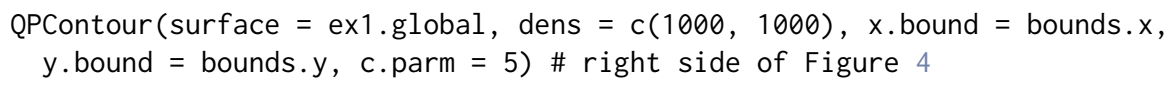

QPContour() is based on the filled.contour() function from the base package graphics. In most cases, the mesh sizes used for the quasi-potential calculation will be much finer than what is required for useful visualization. The argument dens within QPContour() reduces the points used in the graphics generation. Although it might seem wasteful to perform the original calculations at a mesh size that is finer than the final visualization, this is not so. Choosing the mesh size in the original calculations to be very fine reduces the propagation of errors in the ordered upwind algorithm, and hence leads to a more accurate numerical solution.

An additional option allows users to specify contour levels. R's default for the contour() function creates contour lines that are equally spaced over the range of values specified by the user. In some cases, however, it is desirable to use a non-equidistant spacing for the contours. For example, equallyspaced contours will not capture the topography at the bottom of a basin if the changes in height are much smaller than in other regions in the plot. Simply increasing the number of equally-spaced contour lines does not solve this problem, because steep areas of the plot become completely saturated with lines. QPContour() has a function for non-equidistant contour spacing that condenses contour lines at the bottoms of basins. Specifically, for $n$ contour lines, this function generates a list of contour levels, $\left\{v_{i}\right\}_{i=1}^{n}$, specified by:

$$
v_{i}=\max (\Phi)\left(\frac{i-1}{n-1}\right)^{c}
$$

$c=1$ yields evenly-spaced contours. As $c$ increases, the contour lines become more concentrated near basin bottoms. Figure 4 shows equal contour lines (left panel) and contour lines that are concentrated at the bottom of the basin (right panel, c. parm = 5).

Finally, creating a 3D plot can be very useful for visualizing the features of more complex surfaces. This is especially helpful when considering the physical metaphor of a ball rolling on a surface specified by a quasi-potential (Nolting and Abbott, 2016). R has several packages for 3D plotting, including static plotting with the base function persp() and with the package plot3D (Soetaert, 2014). Interactive plotting is provided by rgl (Adler et al., 2015). To create an interactive 3D plot for Example 1 using rgl, use the code: $\operatorname{persp} 3 d(x=$ ex 1 .global $)$. Figure 5 shows a $3 D$ plot of example 1 using persp3D $(z=$ ex1.global) in plot3D that clearly illustrates the differences between the two local basins. Users can also export the matrix of quasi-potential values and create 3D plots in other programs. 


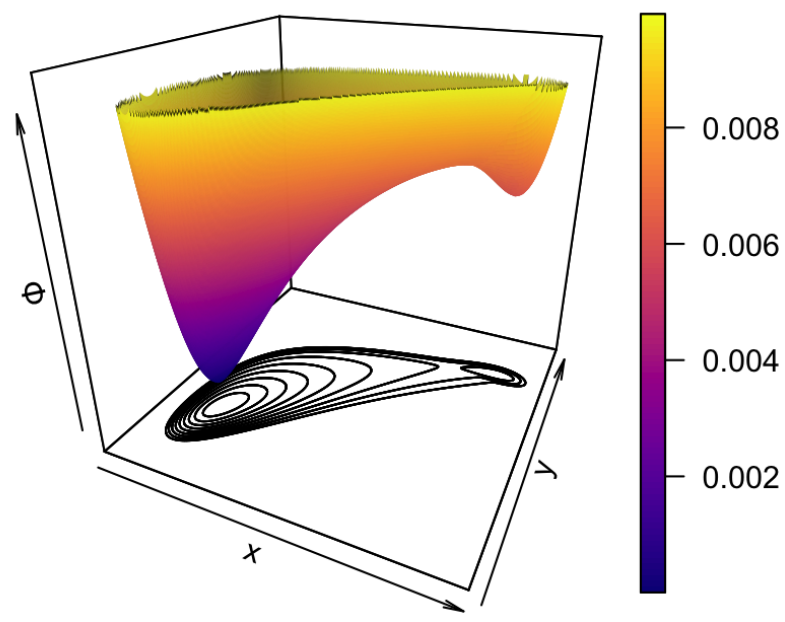

Figure 5: A 3D plot of the of the quasi-potential of System (3) using persp3D() in package plot3D. 3D plotting can further help users visualize the quasi-potential surfaces. Plot colors are generated from package viridis by setting col $=\operatorname{viridis}(n=100$, option $=" A ")$ and contour $=$ TRUE.

\section{Step 6: Vector field decomposition}

Recall that the deterministic skeleton (1) can be visualized as a vector field, as shown in Figure 1. In gradient systems, this vector field is completely determined by the potential function, $V(x, y)$. The name gradient system refers to the fact that the vector field is the negative of the potential function's gradient,

$$
\left[\begin{array}{l}
f_{1}(x, y) \\
f_{2}(x, y)
\end{array}\right]=-\nabla V(x, y)=-\left[\begin{array}{l}
\frac{\partial V}{\partial x}(x, y) \\
\frac{\partial V}{\partial y}(x, y)
\end{array}\right] .
$$

In non-gradient systems, the vector field can no longer be represented solely in terms of the gradient of $\Phi(x, y)$. Instead, there is a remainder component of the vector field, $\mathbf{r}(x, y)=\left[\begin{array}{l}r_{1}(x, y) \\ r_{2}(x, y)\end{array}\right]$. The vector field can be decomposed into two terms:

$$
\left[\begin{array}{l}
f_{1}(x, y) \\
f_{2}(x, y)
\end{array}\right]=-\nabla \Phi(x, y)+\mathbf{r}(x, y)=-\left[\begin{array}{l}
\frac{\partial \Phi}{\partial x}(x, y) \\
\frac{\partial \Phi}{\partial y}(x, y)
\end{array}\right]+\left[\begin{array}{l}
r_{1}(x, y) \\
r_{2}(x, y)
\end{array}\right] .
$$

The remainder vector field is orthogonal to the gradient of the quasi-potential everywhere. That is, for every $(x, y)$ in the domain,

$$
\nabla \Phi(x, y) \cdot \mathbf{r}(x, y)=0 .
$$

An explanation of this property can be found in Nolting and Abbott (2016).

The remainder vector field can be interpreted as a force that causes trajectories to circulate around level sets of the quasi-potential. QPot enables users to perform this decomposition. The function VecDecomAll() calculates the vector field decomposition, and outputs three vector fields: the original deterministic skeleton, $\mathbf{f}(x, y)$; the gradient vector field, $-\nabla \Phi(x, y)$; and the remainder vector field, $\mathbf{r}(x, y)$. Each of these three vector fields can be output alone using $\operatorname{VecDecomVec}(), \operatorname{VecDecomGrad}()$, or VecDecomRem(). These vector fields can be visualized using the function VecDecomPlot(). Code to create the vector fields from VecDecomAll() is displayed below; code for generating individual vector fields can be found in the man pages accessible by help() for $\operatorname{VecDecomVec}(), \operatorname{VecDecomGrad}()$, or VecDecomRem(). The gradient and remainder vector fields are shown in the left and right columns of Figure 6, respectively, with proportional vectors (top row) and equal-length vectors (bottom row). Three arguments within VecDecomPlot() are important to creating comprehensible plots: dens, tail.length, and head.length. The dens parameter specifies the number of arrows in the plot window along the $x$ and $y$ axes. The argument tail. length scales the length of arrow tails. The argument head. length scales the length of arrow heads. The function arrows() makes up the base of VecDecomplot(), and arguments can be passed to it, as well as to plot(). The code below produces all 
three vector fields from the multi-dimensional array returned by VecDecomAll():

\#\# Calculate all three vector fields.

VDAll <- VecDecomAll (surface = ex1.global, x.rhs = parms.eqn.x, y.rhs = parms.eqn.y,

$\mathrm{x}$. bound $=$ bounds. $\mathrm{x}, \mathrm{y} \cdot$ bound $=$ bounds.y)

\#\# Plot the deterministic skeleton vector field.

$\operatorname{VecDecomPlot}(\mathrm{x} . \mathrm{field}=\operatorname{VDAll}[,, 1], \mathrm{y}$. field $=\operatorname{VDAll}[, 2]$, dens $=c(25,25)$,

$\mathrm{x}$. bound $=$ bounds. $x, y \cdot$ bound $=$ bounds.y, $x \lim =c(0,11), y \lim =c(0,6)$,

arrow. type $=$ "equal", tail.length $=0.25$, head.length $=0.025$ )

\#\# Plot the gradient vector field.

VecDecomPlot $(x$. field $=\operatorname{VDAll}[$, , 3], y.field $=\operatorname{VDAll}[,, 4]$, dens $=c(25,25)$,

$\mathrm{x}$. bound $=$ bounds. $\mathrm{x}, \mathrm{y}$. bound $=$ bounds.y, arrow.type = "proportional",

tail.length $=0.25$, head.length $=0.025$ )

\#\# Plot the remainder vector field.

VecDecomPlot $(x$. field $=\operatorname{VDAll}[$, , 5], y.field $=\operatorname{VDAll}[,, 6]$, dens $=c(25,25)$, $\mathrm{x}$. bound $=$ bounds. $\mathrm{x}, \mathrm{y}$. bound = bounds.y, arrow.type = "proportional",

tail. length $=0.35$, head. length $=0.025$ )
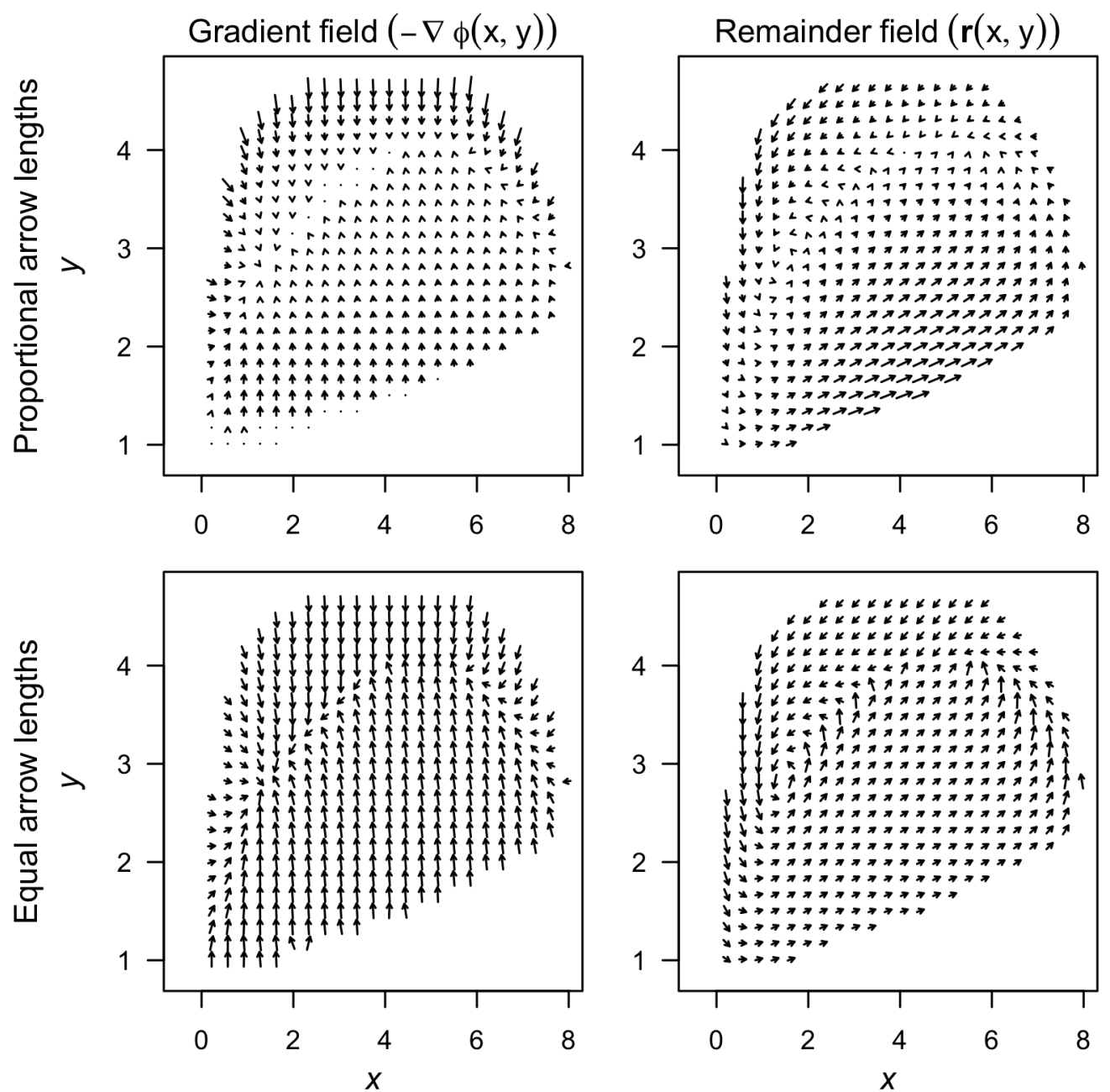

Figure 6: The gradient (left column) and remainder (right column) fields, plotted with arrow. type $=$ "proportional" (top row) and arrow.type = "equal" (bottom row) arrow lengths using VecDecomPlot() for System (3). 


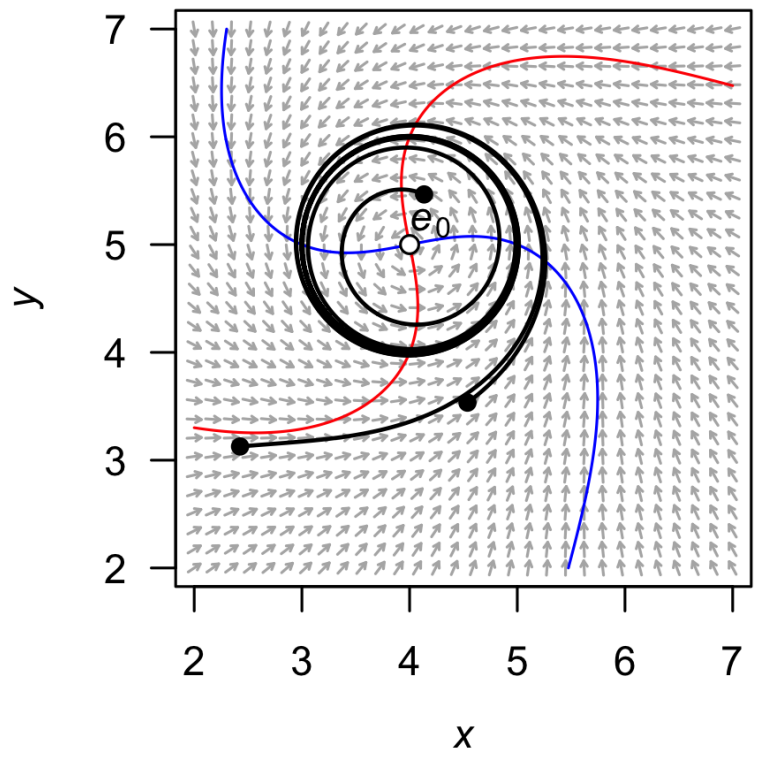

Figure 7: A stream plot of the deterministic skeleton of System (4). The blue line is an $x$-nullcline (where $\frac{d x}{d t}=0$ ) and the red line is a $y$-nullcline (where $\frac{d y}{d t}=0$ ). The open circle is an unstable equilibrium. Particular solutions are shown as black lines, with filled circles as initial conditions. Made using the package phaseR.

\section{Example 2: A model with a limit cycle}

Consider the following model:

$$
\begin{aligned}
& d X(t)=\left(-(Y(t)-\beta)+\mu(X(t)-\alpha)\left(1-(X(t)-\alpha)^{2}-(Y(t)-\beta)^{2}\right)\right) d t+\sigma d W_{1}(t) \\
& d Y(t)=\left((X(t)-\alpha)+\mu(Y(t)-\beta)\left(1-(X(t)-\alpha)^{2}-(Y(t)-\beta)^{2}\right)\right) d t+\sigma d W_{2}(t) .
\end{aligned}
$$

This model will demonstrate QPot's ability to handle limit cycles. We will analyze this example with $\mu=0.2, \alpha=4$, and $\beta=5$.

\section{Step 1: Analyzing the deterministic skeleton}

The deterministic skeleton of this system has one equilibrium, $\mathbf{e}_{0}=(4,5)$, which is an unstable spiral point. Figure 7 shows a stream plot of the deterministic skeleton of System (4). A particular solution of the deterministic skeleton of System (4) can be found using rootSolve and deSolve. The stream plot and a few particular solutions suggest that there is a stable limit cycle. To calculate the limit cycle, one can find a particular solution over a long time interval (e.g., Figure 7 has three trajectories run for $T=100$ ). The solution will eventually converge to the limit cycle. One can drop the early part of the trajectory until only the closed loop of the limit cycle remains. There are more elegant ways to numerically find a periodic orbit (even when those orbits are unstable). For more information on these methods, see Chua and Parker (1989). In this example, the limit cycle is shown by the thick black line in Figure 7. For calculation of the quasi-potential, it is sufficient to input a single point that lies on the limit cycle. For this example, one such point is $\mathbf{z}=(4.15611,5.98774)$.

\section{Step 2: Stochastic simulation}

Figures 8 and 9a show a time series for a realization of (4) with $\sigma=0.1, \Delta t=5 \times 10^{-3}, T=2500$ and initial condition $\left(x_{0}, y_{0}\right)=(3,3)$. Figure $9 \mathrm{~b}$ shows a density plot of a realization with the same parameters, except $T=2.5 \times 10^{3}$.

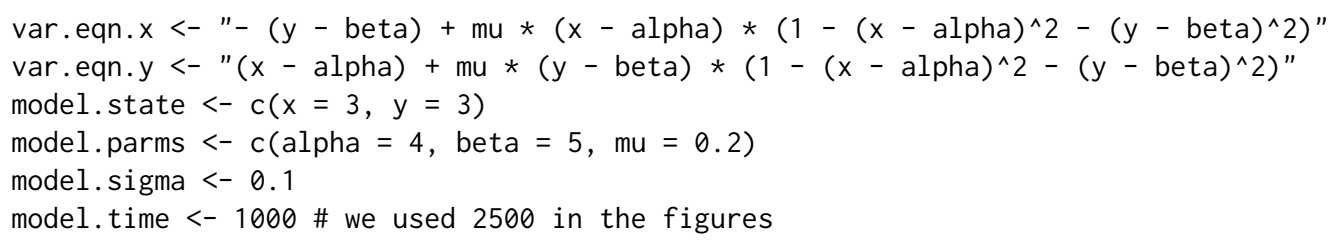




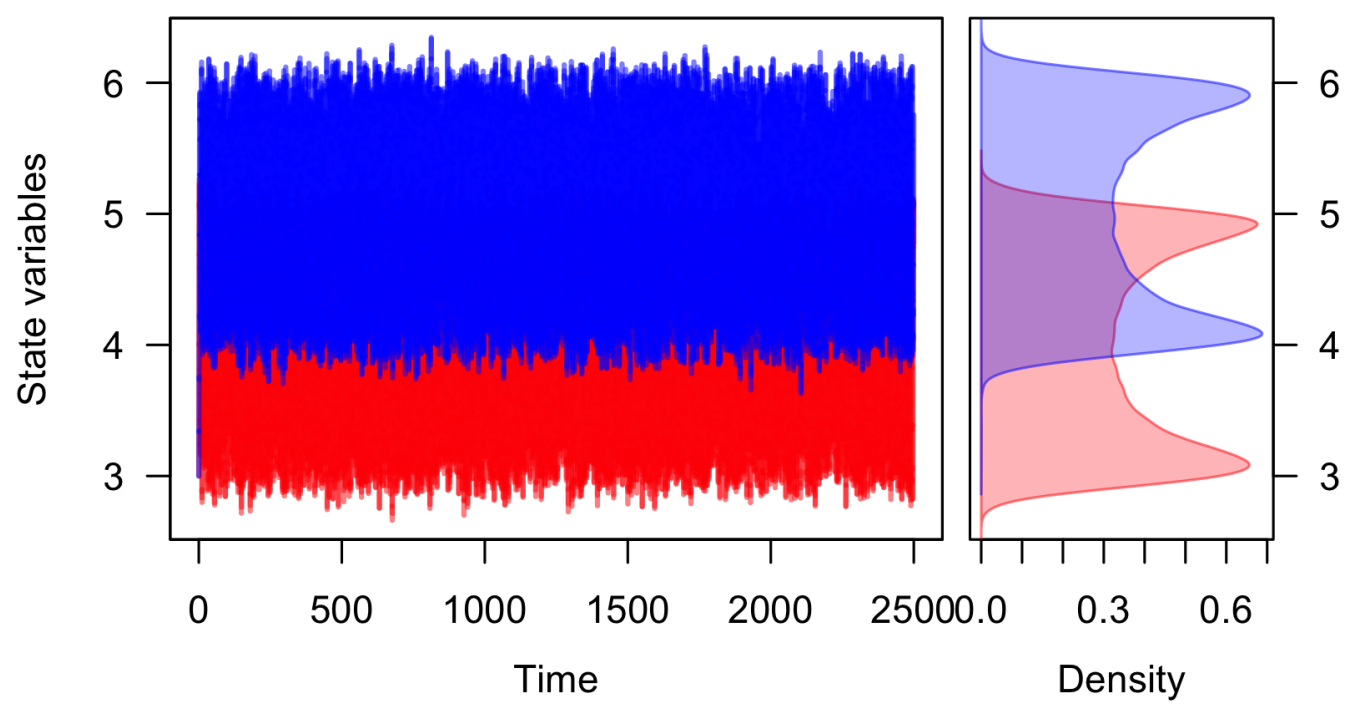

Figure 8: A realization of System (4) created using TSPlot(), with $x$ in blue and $y$ in red. The left side of (a) shows the time series. The right side of (a), which is enabled with the default dens = TRUE, shows a histogram of the $x$ and $y$ values over the entire realization.

model. deltat $<-0.005$

ts.ex2 <- TSTraj $(y 0=$ model.state, time = model.time, deltat $=$ model.deltat,

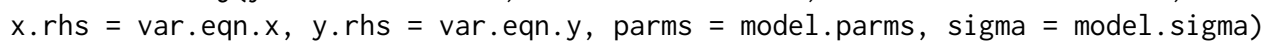

TSPlot (ts.ex2, deltat $=$ model. deltat)

\# Figure 8

TSPlot (ts.ex2, deltat $=$ model.deltat, $\operatorname{dim}=2$, line.alpha $=25$ ) \# Figure 9 a

TSDensity (ts.ex2, $\operatorname{dim}=1$ )

TSDensity (ts.ex2, dim $=2$ )

\# Histogram

\# Figure 9b
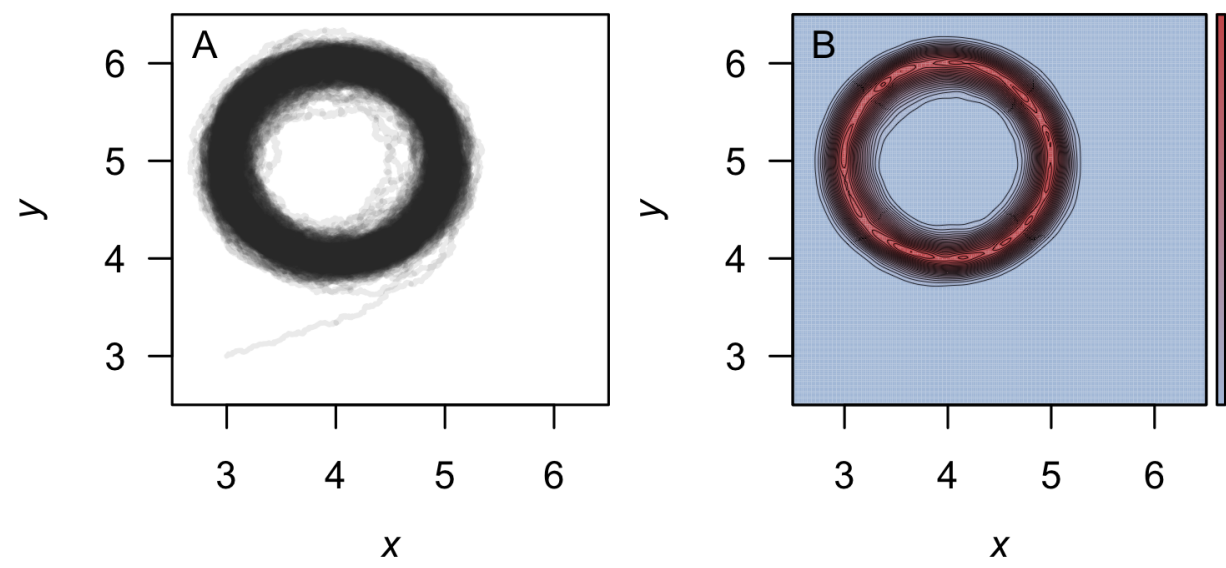

0.12

0.10

0.08

0.06

0.04

0.02

0.00

Figure 9: (A) The realization of System (4) plotted in $(x, y)$-space (dim $=2$ in the function TSPlot ()$)$ (B) A density plot obtained from a realization of System (4) using TSDensity() with dim $=2$. Red corresponds to high density, and blue to low density.

\section{Step 3: Local quasi-potential calculation}

In this example, there are no stable equilibrium points. There is one stable limit cycle, and this can be used to obtain a local quasi-potential. Using $\mathbf{z}$ as the initial point for the ordered-upwind algorithm and $L x_{1}=-0.5, L y_{1}=-0.5, L x_{2}=7.5, L y_{2}=7.5, N_{x}=4000$ and $N_{y}=4000$, one obtains a local quasi-potential, $\Phi_{\mathbf{z}}(x, y)$. The following code generates the local quasi-potential $\Phi_{\mathbf{z}}(x, y)$ : 

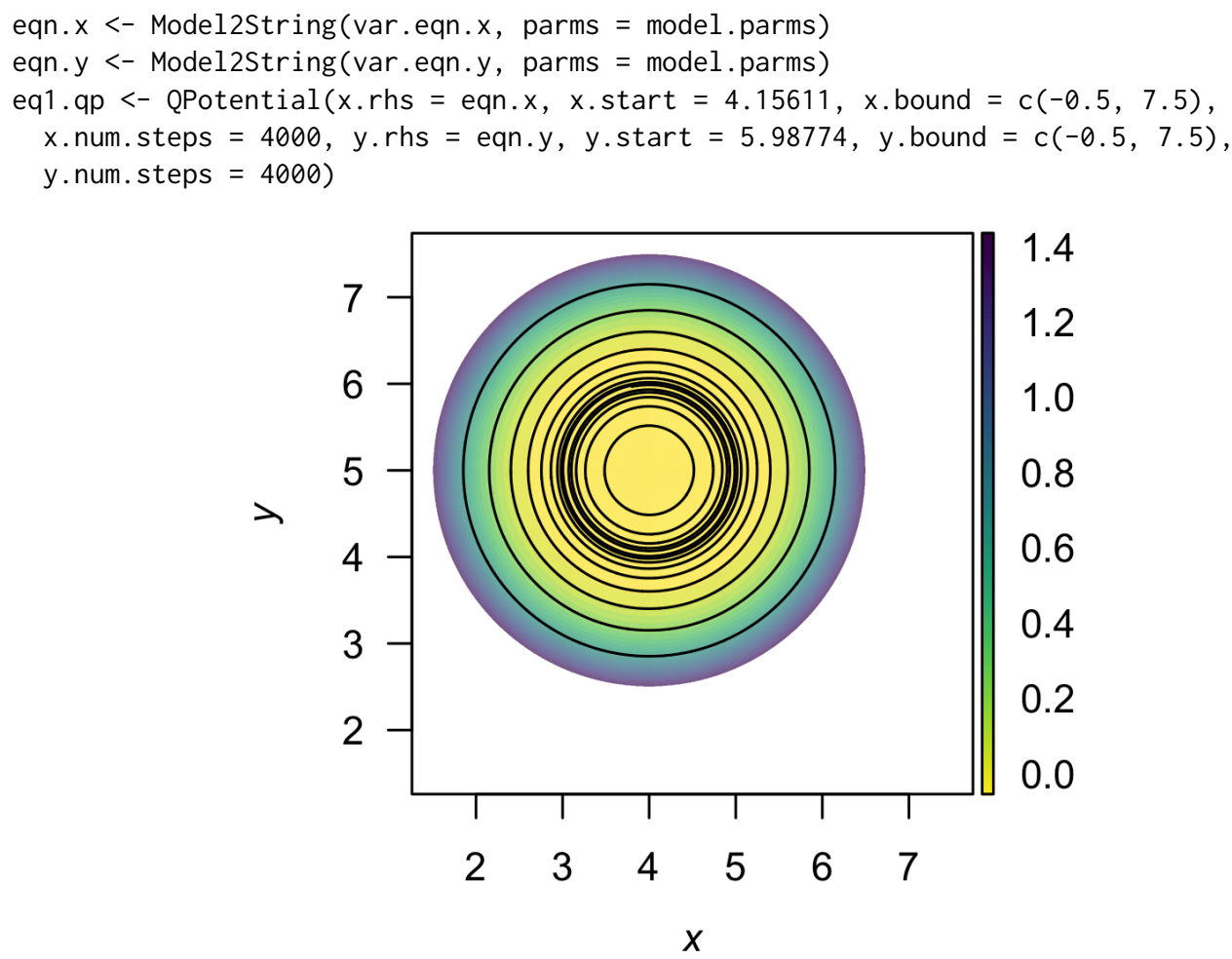

Figure 10: A contour plot of the quasi-potential of System (4) using QPContour (). Yellow corresponds to low values of the quasi-potential, and purple to high values.

\section{Step 4: Global quasi-potential calculation}

There is only one local quasi-potential in this example, so it is the global quasi-potential, $\Phi(x, y)=$ $\Phi_{\mathbf{z}}(x, y)$.

\section{Step 5: Global quasi-potential visualization}

Figure 10 shows a contour plot of the global quasi-potential.

QPContour (eq1.qp, dens $=c(1000,1000), x$. bound $=c(-0.5,7.5)$,

$y \cdot$ bound $=c(-0.5,7.5), c \cdot$ parm $=10)$

\section{Example 3: More complicated local quasi-potential pasting}

In Example 1, the procedure for pasting local quasi-potentials together into a global quasi-potential was a simple, two-step process. First, one of the local quasi-potentials was translated so that the two surfaces agreed at the saddle point separating the two basins of attraction. Second, the global quasi-potential was obtained by taking the minimum of the two surfaces at each point. A general algorithm for pasting local quasi-potentials, as explained in Graham and Tél (1986) and Roy and Nauman (1995), is slightly more complicated. This process is automated in QPGlobal(), but it is worth understanding the process in order to correctly interpret the outputs.

To understand the full algorithm, consider the following model:

$$
\begin{aligned}
& d X(t)=X(t)\left(\left(1+\alpha_{1}\right)-X(t)^{2}-X(t) Y(t)-Y(t)^{2}\right) d t+\sigma d W_{1}(t) \\
& d Y(t)=Y(t)\left(\left(1+\alpha_{2}\right)-X(t)^{2}-X(t) Y(t)-Y(t)^{2}\right) d t+\sigma d W_{2}(t)
\end{aligned}
$$

For this analysis, let $\alpha_{1}=1.25$ and $\alpha_{2}=2$. We have selected this model because it demonstrates how QPot can handle an exceptionally tricky global quasi-potential construction. 


\section{Step 1: Analyzing the deterministic skeleton}

The deterministic skeleton of this system has five equilibria. These are $\mathbf{e}_{u 1}=(0,0), \mathbf{e}_{s 1}=(0,-1.73205)$, $\mathbf{e}_{s 2}=(0,1.73205), \mathbf{e}_{u 2}=(-1.5,0)$ and $\mathbf{e}_{u 3}=(1.5,0)$. The eigenvalue analysis shows that $\mathbf{e}_{u 1}$ is an unstable node, $\mathbf{e}_{s 1}$ and $\mathbf{e}_{s 2}$ are stable nodes, $\mathbf{e}_{u 2}$ and $\mathbf{e}_{u 3}$ are saddles. Figure 11 shows a stream plot of the deterministic skeleton of (5). The basin of attraction for $\mathbf{e}_{s 1}$ is the lower half-plane, and the basin of attraction for $\mathbf{e}_{s 2}$ is the upper half-plane.

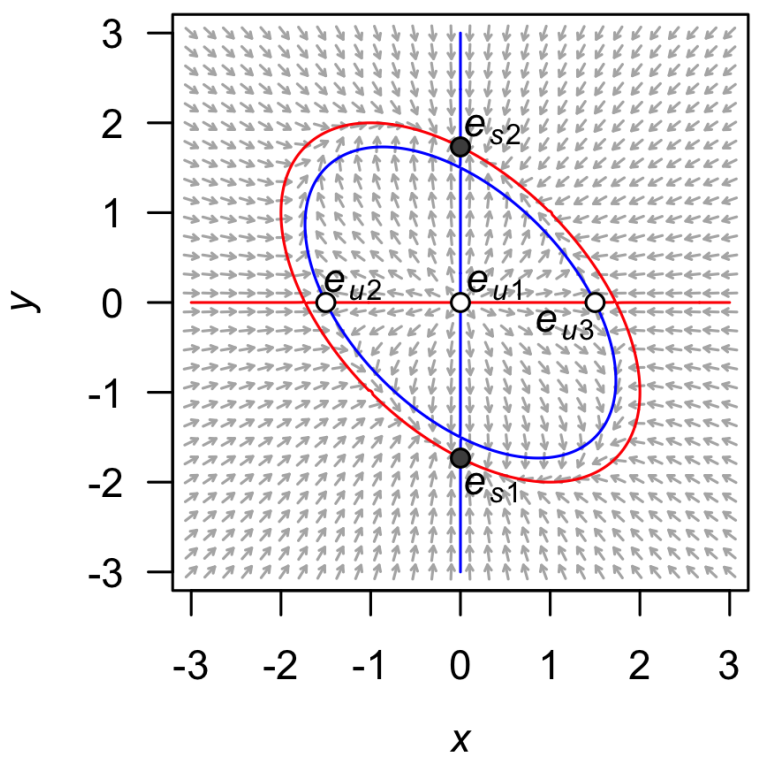

Figure 11: A stream plot of the deterministic skeleton of System (5). The blue line is an $x$-nullcline (where $\frac{d x}{d t}=0$ ) and the red line is a $y$-nullcline (where $\frac{d y}{d t}=0$ ). Open circles are stable equilibria and filled circles are unstable equilibria. Made using the package phaseR.

\section{Step 2: Stochastic simulation}

Figures 12 and 13a show a time series for a realization of System (5) with $\sigma=0.8, \Delta t=0.01, T=5000$ and initial condition $\left(x_{0}, y_{0}\right)=(0.5,0.5)$. Figure $13 \mathrm{~b}$ shows a density plot of this realization.

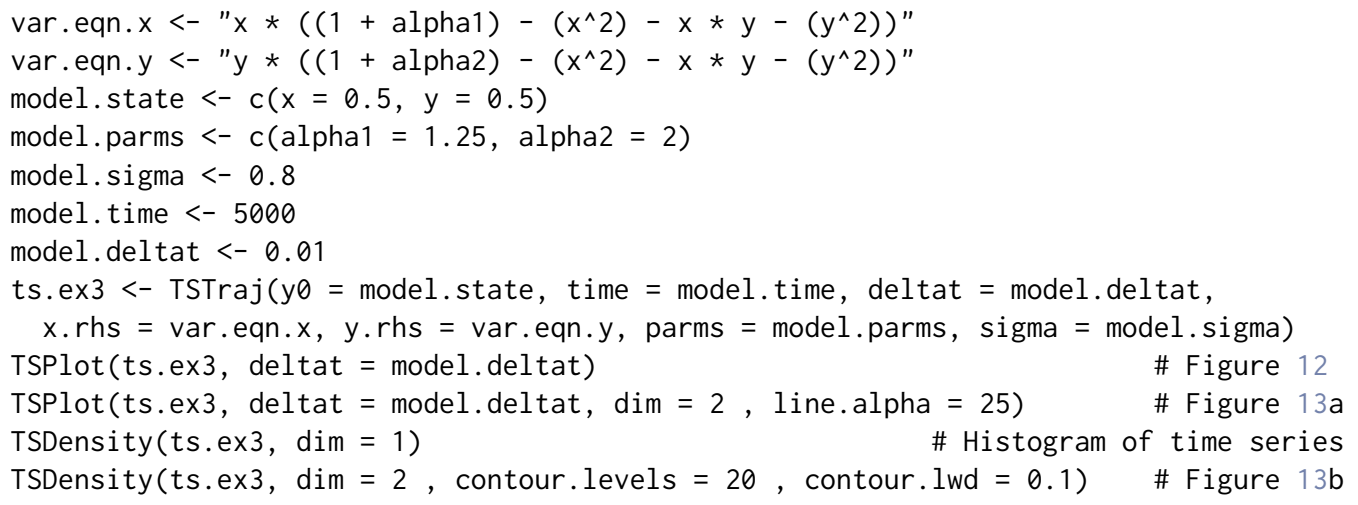

\section{Step 3: Local quasi-potential calculation}

Two local quasi-potentials need to be calculated, $\Phi_{1}(x, y)$ corresponding to $\mathbf{e}_{s 1}$, and $\Phi_{2}(x, y)$ corresponding to $\mathbf{e}_{s 2}$. In both cases, sensible boundary and mesh choices are $L x_{1}=-3, L y_{1}=-3, L x_{2}=3$, $L y_{2}=3, N_{x}=6000$, and $N_{y}=6000$. 


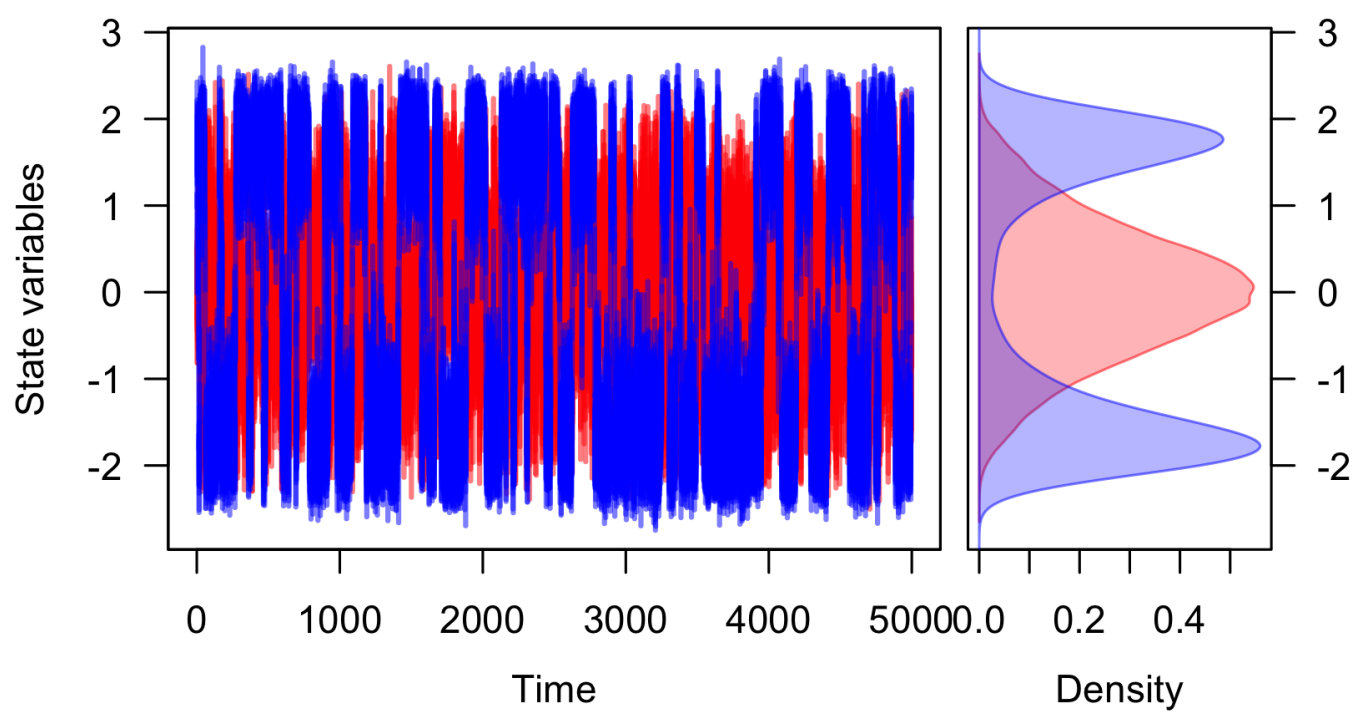

Figure 12: A realization of System (5) created using TSPlot(), with $x$ in blue and $y$ in red. The left panel shows the time series. The right panel, which is enabled by default with parameter dens = TRUE in the function TSPlot(), shows a histogram of the $x$ and $y$ values over the entire realization.
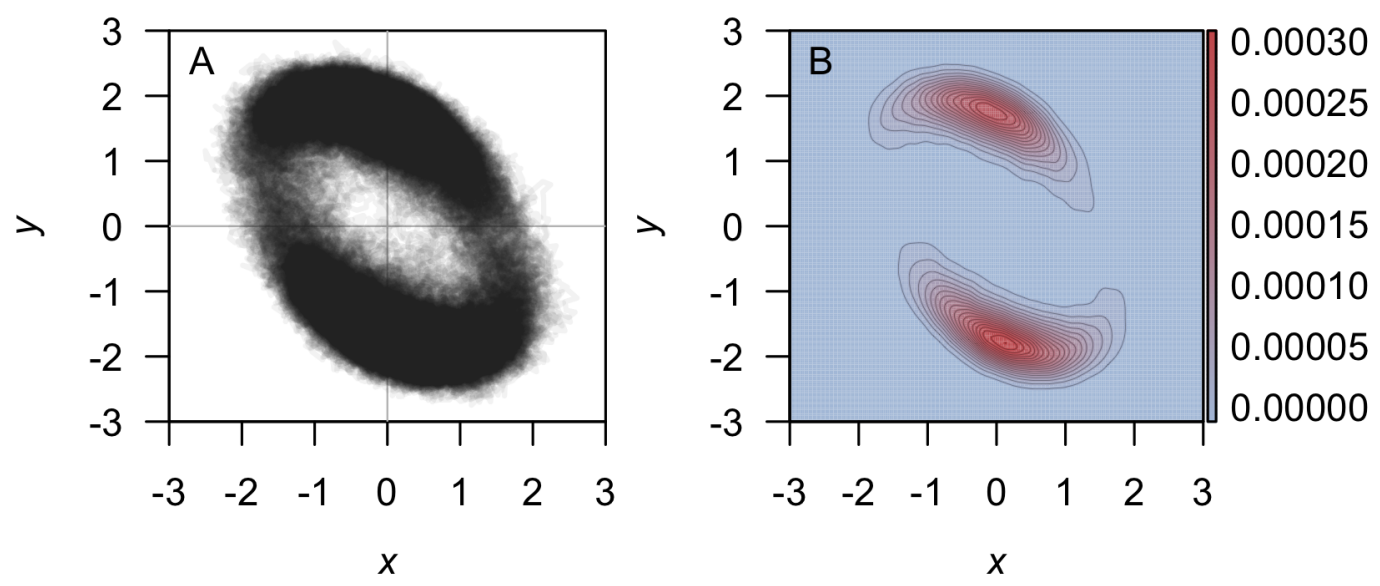

Figure 13: (A) The realization of System (5) plotted in $(x, y)$-space with TSPlot () with dim = 2. (B) A density plot obtained from the realization of System (5) by using the function TSDensity () with dim = 2 , contour. levels $=20$, and contour. $1 w d=0.1$. Red corresponds to high density, and blue to low density.

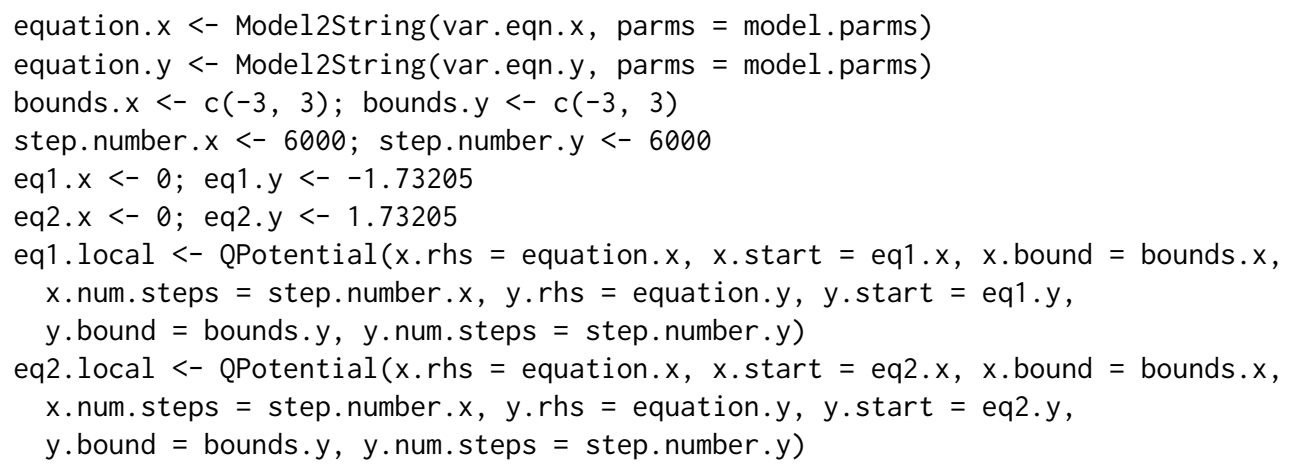

\section{Step 4: Global quasi-potential}

If one were to naively try to match the local quasi-potentials at $\mathbf{e}_{u 2}$, then they would not match at $\mathbf{e}_{u 3}$, and vice versa. To overcome this problem, it is necessary to think more carefully about how trajectories transition between basins of attraction. This issue can be dealt with rigorously (Graham 
and Tél, 1986; Roy and Nauman, 1995), but the general principles are outlined here. Let $\Omega_{1}$ be the basin of attraction corresponding to $\mathbf{e}_{s 1}$ and $\Omega_{2}$ be the basin of attraction corresponding to $\mathbf{e}_{s 2}$. Let $\partial \Omega$ be the separatrix between these two basins (i.e., the $x$-axis). The most probable way for a trajectory to transition from $\Omega_{1}$ to $\Omega_{2}$ involves passing through the lowest point on the surface specified by $\Phi_{1}$ along $\partial \Omega$. Examination of $\Phi_{1}$ indicates that this point is $\mathbf{e}_{u 2}$. In the small-noise limit, the transition rate from $\Omega_{1}$ to $\Omega_{2}$ will correspond to $\Phi_{1}\left(\mathbf{e}_{u 2}\right)$. Similarly, the transition rate from $\Omega_{2}$ to $\Omega_{1}$ will correspond to $\Phi_{2}\left(\mathbf{e}_{u 3}\right)$. The transition rate into $\Omega_{1}$ must equal the transition rate out of $\Omega_{2}$. Therefore, the two local quasi-potentials should be translated so that the minimum heights along the separatrix are the same. In other words, one must define translated local quasi-potentials $\Phi_{1}^{*}(x, y)=\Phi_{1}(x, y)+c_{1}$ and $\Phi_{2}^{*}(x, y)=\Phi_{2}(x, y)+c_{2}$ so that

$$
\min \left(\Phi_{1}^{*}(x, y) \mid(x, y) \in \partial \Omega\right)=\min \left(\Phi_{2}^{*}(x, y) \mid(x, y) \in \partial \Omega\right) .
$$

In Example 1, the minima of both local quasi-potentials occurred at the same point, so the algorithm amounted to matching at that point. In Example 3, the minimum saddle for $\Phi_{1}$ is $\mathbf{e}_{u 2}$ and the minimum saddle for $\Phi_{2}$ is $\mathbf{e}_{u 3}$; the heights of the surfaces at these respective points should be matched. Thus, $c_{1}=\Phi_{2}\left(e_{u 3}\right)-\Phi_{1}\left(e_{u 3}\right)$ and $c_{2}=\Phi_{1}\left(e_{u 2}\right)-\Phi_{2}\left(e_{u 2}\right)$. Conveniently in Example 3, this is satisfied without requiring any translation (one can use $c_{1}=c_{2}=0$ ). Finally, the global quasi-potential is found by taking the minimum value of the matched local quasi-potentials at each point. This process is automated in QPot, but users can also manipulate the local quasi-potential matrices manually to verify the results. This is recommended when dealing with unusual or complicated separatrices. The code below applies the automated global quasi-potential calculation to Example 3.

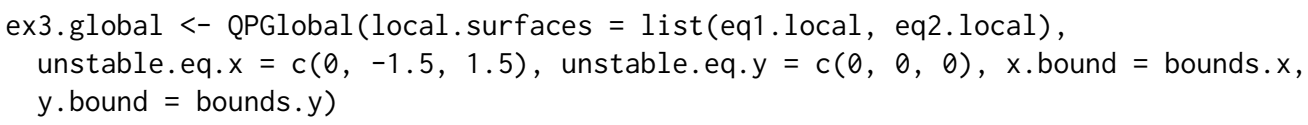

\section{Step 5: Global quasi-potential visualization}

Figure 14 shows a contour plot of the global quasi-potential. Note that the surface is continuous, but not smooth. The lack of smoothness is a generic feature of global quasi-potentials created from pasting local quasi-potentials. Cusps usually form when switching from the part of solution obtained from one local quasi-potential to the other.
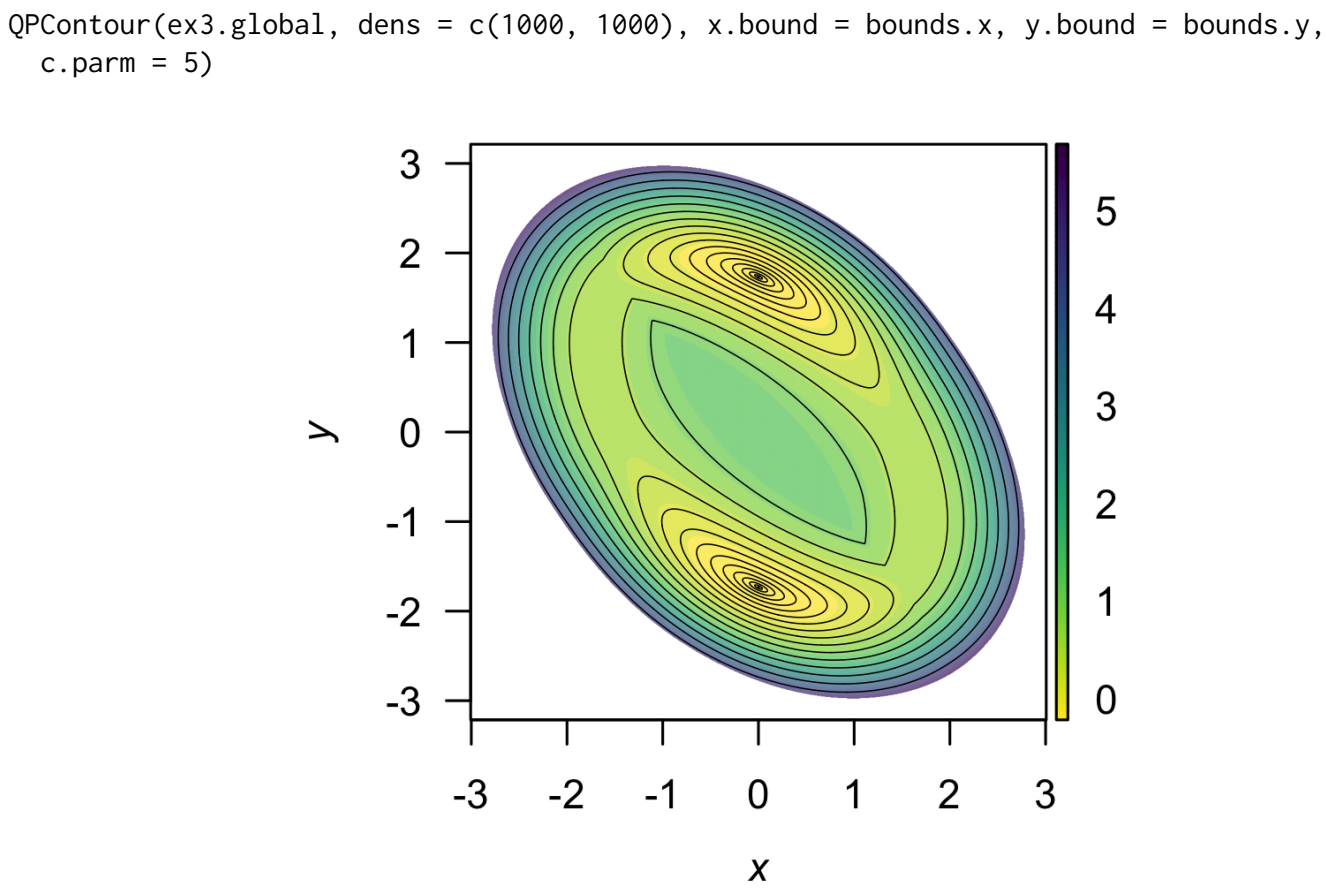

Figure 14: A contour plot of the quasi-potential of System (5) using the function QPContour (). Yellow corresponds to low values of the quasi-potential, and purple to high values. 


\section{Boundary behavior}

It is important to consider the type of behavior that should be enforced at the boundaries and on coordinate axes $(x=0$ and $y=0)$. By default, the ordered-upwind method computes the quasipotential for the system defined by the user, without regard for the influence of the boundaries or the significance of these axes. In some cases, however, a model is only valid in a subregion of phase space. For example, in many population models, only the non-negative phase space is physically meaningful. In such cases, it is undesirable to allow the ordered-upwind method to consider trajectories that pass through negative phase space. In the default mode for QPotential(), if $(x, y)$ lies in positive phase space, $\Phi(x, y)$ can be impacted by the vector field in negative phase space, if the path corresponding to the minimum work passes through negative phase space. The argument bounce $=$ " $d$ " corresponds to this (d)efault behavior. A user can prevent the ordered upwind method from passing trajectories through negative phase space by using the option bounce = "p" for (p)ositive values only. This option can be interpreted as a reflecting boundary condition. It forces the front of solutions obtained by the ordered upwind method to stay in the defined boundaries, which is positive phase space in this case. A more generic option is bounce = " $b$ " for (b)ounce, which allows users to supply reflecting boundaries other than the coordinate axes. These are set with $\mathrm{x}$. bound and y.bound. Small numerical errors at a reflecting boundary can cause the algorithm to terminate prematurely. To avoid this, the option bounce. edge adds a small amount of padding between the reflecting boundary and the edge of the computational domain.

\section{Different noise terms}

In the cases considered so far, the noise terms for the $X$ and $Y$ variables have had identical intensity. This was useful for purposes of illustration in the algorithm, but it will often be not true for real-world systems. Fortunately, QPot can accommodate other noise terms with coordinate transforms. Consider a system of the form:

$$
\begin{aligned}
& d X(t)=f_{1}(X(t), Y(t)) d t+\sigma g_{1} d W_{1}(t) \\
& d Y(t)=f_{2}(X(t), Y(t)) d t+\sigma g_{2} d W_{2}(t) .
\end{aligned}
$$

$\sigma$ is a scaling parameter that specifies the overall noise intensity. The parameters $g_{1}$ and $g_{2}$ specify the relative intensity of the two noise terms. To transform this system into a form that is usable for QPot, make the change of variable $\tilde{X}=g_{1}^{-1} X$ and $\tilde{Y}=g_{2}^{-1} Y$. In the new coordinates, the drift terms (that is, the terms multiplied by $d t$ ), are different. These are $\tilde{f}_{1}(\tilde{X}, \tilde{Y})=g_{1}^{-1} f_{1}\left(g_{1} \tilde{X}, g_{2} \tilde{Y}\right)$ and $\tilde{f}_{2}(\tilde{X}, \tilde{Y})=g_{2}^{-1} f_{2}\left(g_{1} \tilde{X}, g_{2} \tilde{Y}\right)$. These new drift terms should be used as the deterministic skeleton that is input into QPot. After obtaining the global quasi-potential for these transformed coordinates, one can switch back to the original coordinates for plotting.

Many models contain multiplicative noise terms. These are of the form:

$$
\begin{aligned}
& d X(t)=f_{1}(X(t), Y(t)) d t+\sigma g_{1} X(t) d W_{1}(t) \\
& d Y(t)=f_{2}(X(t), Y(t)) d t+\sigma g_{2} Y(t) d W_{2}(t) .
\end{aligned}
$$

To transform this system into a form that is usable for QPot, make the change of variable $\tilde{X}=g_{1}^{-1} \ln (X)$ and $\tilde{Y}=g_{2}^{-1} \ln (Y)$. This is called the Lamperti transform (Iacus, 2009). It is not always possible to transform a multidimensional stochastic differential equation with multiplicative noise into one with additive noise (Pavliotis, 2014), but in special cases like (7) it is. This coordinate change is non-linear, so Itô's lemma introduces extra terms into the drift of the transformed equations. If $\sigma$ is small, though, these terms can be discounted, and the new drift terms will remain independent of $\sigma$. These new drift terms can be input into QPot. After obtaining the global quasi-potential for these transformed coordinates, one can switch back to the original coordinates.

\section{Conclusion}

QPot is an R package that provides several important tools for analyzing two-dimensional systems of stochastic differential equations. Future efforts will work toward extending QPot to higherdimensional systems, but this is a computationally challenging task. QPot includes functions for generating realizations of the stochastic differential equations, and for analyzing and visualizing the results. A central component of QPot is the calculation of quasi-potential functions, which are highly useful for studying stochastic dynamics. For example, quasi-potential functions can be used to compare the stability of different attractors in stochastic systems, a task that traditional linear stability 
analysis is poorly suited for (Nolting and Abbott, 2016). By offering an intuitive way to quantify attractor stability, quasi-potentials are poised to become an important means of understanding phenomena like metastability and alternative stable states. QPot makes quasi-potentials accessible to R users interested in applying this new framework.

\section{Author contributions}

K.C.A, C.M.M., B.C.N., and C.R.S. designed the project. M.K.C. wrote the C code for finding the quasi-potential; C.M.M. and C.R.S. wrote the R code and adapted the C code.

\section{Acknowledgments}

This work was supported by a Complex Systems Scholar grant to K.C.A. from the James S. McDonnell Foundation. M.K.C. was partially supported by NSF grant 1217118 . We also thank the anonymous reviewers for their constructive comments and suggestions which helped us to improve the quality of our paper.

\section{Bibliography}

D. Adler, D. Murdoch, O. Nenadic, S. Urbanek, M. Chen, A. Gebhardt, B. Bolker, G. Csardi, A. Strzelecki, and A. Senger. rgl: 3D Visualization Device System for $R$ using OpenGL, 2015. URL https://CRAN.R-project.org/package=rgl. R package version 0.95.1247. [p27]

E. J. Allen. Modeling with Ito Stochastic Differetnial Equations, volume 22 of Mathematical Modelling: Theory and Applications. Springer-Verlag, 2007. [p20]

A. Brouste, M. Fukasawa, H. Hino, S. M. Iacus, K. Kamatani, Y. Koike, H. Masuda, R. Nomura, T. Ogihara, Y. Shimuzu, M. Uchida, and N. Yoshida. The YUIMA project: A computational framework for simulation and inference of stochastic differential equations. Journal of Statistical Software, 57(4):1-51, 2014. doi: 10.18637/jss.v057.i04. [p22]

M. K. Cameron. Finding the quasipotential for nongradient SDEs. Physica D, 241(18):1532-1550, 2012. $[\mathrm{p} 21,24,25]$

T. S. P. L. Chua and T. S. Parker. Practical Numerical Algorithms for Chaotic Systems, chapter 5. SpringerVerlag, 1989. [p30]

J. S. Collie and P. D. Spencer. Modeling predator-prey dynamics in a fluctuating environment. Canadian Journal of Fisheries and Aquatic Sciences, 51(12):2665-2672, 1994. [p21]

M. I. Freidlin and A. D. Wentzell. Random Perturbations of Dynamical Systems, volume 260. SpringerVerlag, 2012. [p21, 26]

S. Garnier. viridis: Default Color Maps from 'matplotlib', 2016. URL https://github.com/sjmgarnier/ viridis. R package version 0.3.4. [p27]

R. Graham and T. Tél. Nonequilibrium potential for coexisting attractors. Physical Review. A, 33(2): 1322-1337, 1986. [p26, 32, 34]

M. J. Grayling. phaseR: Phase Plane Analysis of One and Two Dimensional Autonomous ODE Systems, 2014a. URL https: //CRAN. R-project.org/package=phaseR. R package version 1.3. [p22]

M. J. Grayling. phaseR: An R package for phase plane analysis of autonomous ODE systems. The $R$ Journal, 6(2):43-51, 2014b. [p22]

A. Guidoum and K. Boukhetala. Sim.DiffProc: Simulation of Diffusion Processes., 2016. URL https: //CRAN.R-project.org/package=Sim. DiffProc. R package version 3.2. [p22]

S. M. Iacus. Simulation and Inference for Stochastic Differential Equations: With R Examples, volume 1. Springer Science \& Business Media, 2009. [p20,36]

C. Moore, C. Stieha, B. Nolting, M. Cameron, and K. Abbott. QPot: Quasi-Potential Analysis for Stochastic Differential Equations, 2016. URL https://CRAN.R-project.org/package=QPot, https: //github.com/bmarkslash7/QPot. R package version 1.2. [p19] 
B. C. Nolting and K. C. Abbott. Balls, cups, and quasi-potentials: Quantifying stability in stochastic systems. Ecology, 97(4):850-864, 2016. [p20, 21, 27, 28, 37]

G. A. Pavliotis. Stochastic Processes and Applications: Diffusion Processes, the Fokker-Planck and Langevin Equations, volume 60 of Texts in Applied Mathematics. Springer-Verlag, New York, 2014. [p36]

R. V. Roy and E. Nauman. Noise-induced effects on a non-linear oscillator. Journal of Sound and Vibration, 183(2):269-295, 1995. doi: 10.1006/jsvi.1995.0254. [p26, 32, 35]

J. A. Sethian and A. Vladimirsky. Ordered upwind methods for static Hamilton-Jacobi equations. Proceedings of the National Academy of Sciences, 98(20):11069-11074, 2001. doi: 10.1073/pnas.201222998. [p24]

J. A. Sethian and A. Vladimirsky. Ordered upwind methods for static Hamilton-Jacobi equations: Theory and algorithms. SIAM Journal on Numerical Analysis, 41(1):325-363, 2003. doi: 10.1137/ S0036142901392742. [p24]

K. Soetaert. plot3D: Plotting Multi-Dimentional Data in R, 2014. URL https://CRAN. R-project.org/ package=plot3D. R package version 1.0-2. [p27]

K. Soetaert and P. M. Herman. A Practical Guide to Ecological Modelling: Using R as a Simulation Platform. Springer Science \& Business Media, 2008. [p21]

K. Soetaert, T. Petzoldt, and R. W. Setzer. Solving differential equations in R: Package deSolve. Journal of Statistical Software, 33(9):1-25, 2010. doi: 10.18637/jss.v033.i09. [p22]

J. H. Steele and E. W. Henderson. A simple plankton model. American Naturalist, 117(5):676-691, 1981. [p21]

Christopher M. Moore

Department of Biology

Case Western Reserve University

United States

life.dispersing@gmail.com

Christopher R. Stieha

Department of Biology

Case Western Reserve University

United States

stieha@hotmail.com

Ben C. Nolting

Department of Biology

Case Western Reserve University

United States

Current:

Department of Mathematics and Statistics

California State University, Chico

United States

bnolting@gmail.com

Maria K. Cameron

Department of Mathematics

University of Maryland

United States

cameron@math. umd.edu

Karen C. Abbott

Department of Biology

Case Western Reserve University

United States

kcabbott@case.edu 